\title{
ANÁLISIS Y VALORACIÓN DEL ÁREA DE EDUCACIÓN DEL III PLAN DE ACCIÓN PARA PERSONAS CON DISCAPACIDAD EN LA COMUNIDAD DE MADRID (2012-2015)
}

\section{Action Plan for People with Disabilities in Madrid Autonomous Community (2012-2015). Analysis and evaluation of the Educational Area within this plan}

Gerardo ECHEITA SARRIONANDIA

Universidad Autónoma de Madrid. Facultad de Psicología. Dpto. Interfacultativo de Psicología Evolutiva y de la Educación. Ciudad Universitaria de Cantoblanco. 28049 Madrid gerardo.echeita@uam.es

Cecilia Simón RuedA

Universidad Autónoma de Madrid. Facultad de Psicología. Dpto. Interfacultativo de Psicología Evolutiva y de la Educación

Carmen MÁrquez VÁzQuEZ

Universidad Autónoma de Madrid. Facultad de Educación. Dpto. Interfacultativo de Psicología Evolutiva y de la Educación

María Luz de los Milagros Fernández BlázQueZ

Universidad Autónoma de Madrid. Facultad de Psicología. Dpto. Interfacultativo de Psicología Evolutiva y de la Educación

Elena Pérez de LA Merced

Universidad Autónoma de Madrid. Facultad de Psicología. Dpto. Interfacultativo de Psicología Evolutiva y de la Educación

Amparo Moreno HernándeZ

Universidad Autónoma de Madrid. Facultad de Psicología. Dpto. Interfacultativo de Psicología Evolutiva y de la Educación

Recepción: 17 de febrero de 2017

Fecha de aceptación definitiva: 23 de febrero de 2017 


\section{ANÁLISIS Y VALORACIÓN DEL ÁREA DE EDUCACIÓN DEL III PLAN DE ACCIÓN PARA PERSONAS CON DISCAPACIDAD EN LA COMUNIDAD DE MADRID (2012-2015) \\ G. ECHEITA, C. SIMÓN, C. MÁRQUEZ, M. ${ }^{a}$ L. DE LOS M. FERNÁNDEZ, E. PÉREZ Y A. MORENO}

RESUMEN: Este trabajo presenta los análisis y valoraciones del Área de Educación del Plan de Acción de las Personas con Discapacidad de la Comunidad de Madrid (20122015), realizados tras utilizar la técnica Delphi con un grupo de seis expertos y expertas en educación. El propósito que ha guiado el trabajo ha sido realizar una evaluación formativa del plan implementado, de forma que los análisis generados sean de utilidad tanto para los gestores del próximo plan en proyecto, como para los agentes educativos implicados y, obviamente, para las personas del plan, en tanto que apoyo al ejercicio efectivo de sus derechos. El documento de síntesis elaborado muestra sintéticamente las principales fortalezas de dicho plan, pero, sobre todo, las debilidades o puntos a mejorar. Entre estos cabe señalar la necesidad de una estructuración más eficiente de programas y actuaciones con indicadores precisos de logro, así como la necesidad de replantear modelos y ámbitos de intervención que sean más coherentes con el derecho a una educación inclusiva que ha quedado establecido en la Convención de Derechos de las Personas con Discapacidad y con los modelos de comprensión e intervención para la mejora de la calidad de vida de las personas con discapacidad, entre otros.

Palabras clave: Comunidad de Madrid; personas con discapacidad; evaluación de planes y programas; Técnica Delphi; educación inclusiva; derechos.

AвSTRAct: This paper presents the analysis and assessment of the Education Area of the Plan of Action for Persons with Disabilities in the Autonomous Community of Madrid (2012-2015), carried out after using a Delphi Method with a group of six experts. This work was aimed to make a formative evaluation of the Plan implemented, so that the analysis generated could be used by both managers of the next plan in project, as for educational stakeholders involved in the education field, but obviously, by people with disabilities as well, in order to support them to exercise effectively their rights. The synthesis document shows synthetically the main strengths of the plan but, mostly, the weaknesses or points to improve. Among these should be noted the need of a more efficient structure of programs and actions with clear indicators of achievement. Also there is a need of rethinking current models and areas of intervention, in order to those were much more consistent and coherent with the right to an education inclusive that has been established in the Convention of Rights of People with Disability, and with the models of understanding and intervention to improve their quality of life.

KEY wORDs: Madrid Autonomous Community (Spain); people with disabilities; program evaluation; Delphi Method; inclusive education; rights.

\section{Introducción}

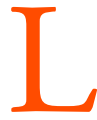

a COMUnidad De MAdRid (CM) en el ejercicio de sus competencias y responsabilidades, al igual que otras comunidades autónomas, viene desarrollando planes sucesivos de actuación en materia de "discapacidad". El objetivo es implementar mejoras sostenidas que incidan significativamente en la calidad de vida (Schalock y Verdugo, 2007) de las personas en situación de discapacidad ${ }^{1}$, y que faciliten

1 En este informe hablamos de personas en situación de discapacidad como un modesto intento de resaltar tres ideas importantes: una, que en primer lugar y sobre todo son “personas” y, por ello, con igual 


\section{ANÁLISIS Y VALORACIÓN DEL ÁREA DE EDUCACIÓN DEL III PLAN DE ACCIÓN PARA PERSONAS CON DISCAPACIDAD EN LA COMUNIDAD DE MADRID (2012-2015) \\ G. ECHEITA, C. SIMÓN, C. MÁRQUEZ, M. ${ }^{a}$ L. DE LOS M. FERNÁNDEZ, E. PÉREZ Y A. MORENO}

el progreso del pleno y satisfactorio ejercicio de sus derechos reconocidos (Naciones Unidas, 2006). Se trata de planes que plantean iniciativas y propuestas en diferentes áreas generales (p. e., salud, empleo, educación, atención temprana, participación social, entre otras) y, en su caso, en otras transversales (p. e., mujer o accesibilidad universal) o de especial preocupación como el área de Trastornos del Espectro Autista.

Sin lugar a dudas, se trata de iniciativas públicas muy necesarias y justas, habida cuenta de las múltiples situaciones de desventaja, discriminación y, por lo tanto, desigualdad que desde antaño viven la inmensa mayoría de personas en dicha situación (niños y niñas, adolescentes, jóvenes o personas adultas). Estos planes son elaborados conforme a los conocimientos, criterios y convicciones del equipo responsable de su diseño, implementación y rendición de cuentas. En este sentido, el III Plan de Acción para Personas con Discapacidad de la Comunidad de Madrid² (PAPCDCM), para el trienio 2012-2015, ha sido diseñado, ejecutado y sus resultados evaluados con los procedimientos, indicadores y participantes que en el mismo se determinaron. Todo ello puede analizarse en la web de la $\mathrm{CM}^{3}$.

El proyecto de investigación Madrid sin Barreras: discapacidad e inclusión social (MADINC), desarrollado con la participación de seis equipos de trabajo vinculados a las Universidades Carlos III, Autónoma de Madrid, Politécnica, Alcalá, Pontificia Comillas de Madrid y UNED, planteó, entre sus objetivos, "llevar a cabo una evaluación de dicho Plan”. Este trabajo presenta los resultados de la evaluación realizada, en este caso, sobre el Área de Educación del citado plan, y generados a través de una técnica Delphi (León y Montero, 2015), adaptada a las finalidades del proyecto en cuestión. En este sentido, y antes de presentar el procedimiento seguido y el informe valorativo generado como resultado de dicha estrategia, parece necesario hacer algunas consideraciones previas sobre todo ello.

\section{Sobre la evaluación de un plan evaluado}

El objetivo de "evaluar el PAPCDCM", en apariencia claro y preciso, debe, no obstante, considerarse en relación con la meta perseguida por el equipo de investigación del proyecto MADINC para que su trabajo tenga sentido y utilidad, en primera

\footnotetext{
dignidad y derechos que el resto de "personas". Segundo, que una de sus singularidades es que se encuentran en una situación de desventaja a consecuencia del resultado de la interacción actual entres sus capacidades y el contexto social, educativo, laboral, etc., en el que se desenvuelven y que se traduce en restricciones en su capacidad de actuar y participar. Pero no por ello dejan de tener "capacidades" como el resto de las personas, algunas más restringidas, pero otras de igual o mayor rango que la mayoría de la población. Se trata de alejarse de la concepción de la "discapacidad" como si fuera un rasgo inherente a la persona y verla como lo que es: una función. Reconocemos, no obstante, que desde el punto de vista científico, profesional y de derechos, el término más aceptado sigue siendo el de Personas con Discapacidad. En todo caso, ambos ponen el énfasis en una visión ecológico-contextual o socioecológica de la discapacidad.

2 http://www.madrid.org/bvirtual/BVCM007242.pdf.

3 http://www.madrid.org/cs/Satellite?c=CM_InfPractica_FA\&cid=1354565353797\& \&idConsejeria= 1109266187278\&idListConsj $=1109265444710 \&$ id Organismo $=1354503014837 \&$ language $=$ es\&pagename $=$ ComunidadMadrid\%2FEstructura\&sm=1109170600517.
} 
ANÁLISIS Y VALORACIÓN DEL ÁREA DE EDUCACIÓN DEL III PLAN DE ACCIÓN PARA PERSONAS CON DISCAPACIDAD EN LA COMUNIDAD DE MADRID (2012-2015)

G. ECHEITA, C. SIMÓN, C. MÁRQUEZ, M. ${ }^{a}$ L. DE LOS M. FERNÁNDEZ, E. PÉREZ Y A. MORENO

instancia, para los responsables de esta tarea en la CM. Pero también para los múltiples y diversos profesionales que, de modo directo o indirecto, intervienen en la educación escolar de los niños y niñas, jóvenes o adultos y, de forma más específica, para las personas en situación de discapacidad de la Comunidad de Madrid, que son sus destinatarias finales y más importantes.

En este sentido, y como hemos anticipado, el propio PAPCDCM contempla y ha ejecutado y difundido una evaluación del mismo, cuyos resultados obran en poder de los responsables de la CM y que han sido públicamente difundidos. En este contexto, el proyecto MADINC no se ha planteado con la finalidad de realizar una evaluación de la evaluación realizada, ni llevar a cabo una que fuera alternativa a esta. Ni el diseño del proyecto MADINC ni los medios de los que el mismo dispone están pensados y son suficientes para ninguna de estas finalidades.

Sin embargo, en un momento en el que, seguramente, la CM estará trabajando (al igual que otras comunidades autónomas, que también lo estarán haciendo en planes similares) en el diseño de un nuevo plan para el siguiente trienio (2016/17-2019/20 o similar), sí creemos que tiene pleno sentido y utilidad hacer aportes que contribuyan a que el siguiente plan mantenga las fortalezas de distinto tipo que ha mostrado el anterior (p. e., en cuestiones de oportunidad, amplitud, apoyo político, participación del movimiento asociativo, etc.), y reduzca las debilidades que, a nuestro juicio, el mismo ha tenido. Finalmente, este análisis puede ser de utilidad para otras iniciativas similares tanto dentro como fuera de España.

Es en el contexto de este objetivo general, que bien cabría denominar de evaluación formativa o para la mejora, en el que han de considerarse las estrategias y actuaciones llevadas a cabo por el equipo de MADINC para "evaluar el PAPCDCM", conforme a los medios, posibilidades y limitaciones del propio proyecto.

Obviamente los resultados que muestran el análisis y la valoración de tales debilidades y fortalezas son discutibles y se han realizado desde los criterios y marcos referenciales adoptados por los miembros del equipo de investigación de este proyecto. Estos pueden no ser compartidos o asumidos por las personas responsables de la planificación, ejecución y evaluación del PAPCDCM o por otras y otros investigadores o actores implicados. Por ello, es necesario hacer explícitos el marco referencial que ha guiado el proceso, la metodología adoptada y, en particular, el método seguido para recabar la información a partir de la cual se han elaborado las conclusiones y sugerencias que aquí se comparten.

\section{Método}

Antes de describir la metodología seguida, es necesario señalar que los análisis que se mostrarán en el apartado correspondiente se han realizado teniendo como marco de referencia el significado y alcance del "derecho a la educación” que recoge la Convención de la ONU sobre los Derechos de las Personas con Discapacidad, en adelante, CDPCD (Naciones Unidas, 2006), junto con el Comentario General n. 4 (CG) que al respecto ha elaborado el Comité de Derechos de las Personas con Discapacidad transcurridos 
ANÁLISIS Y VALORACIÓN DEL ÁREA DE EDUCACIÓN DEL III PLAN DE ACCIÓN PARA PERSONAS CON DISCAPACIDAD EN LA COMUNIDAD DE MADRID (2012-2015)

G. ECHEITA, C. SIMÓN, C. MÁRQUEZ, M. ${ }^{\text {a }}$ L. DE LOS M. FERNÁNDEZ, E. PÉREZ Y A. MORENO

10 años de la Convención (Naciones Unidas, 2016). Estimamos que no hacerlo así vendría a quitar legitimidad a una iniciativa que, como es sabido, cuenta con el aval del sistema de Naciones Unidas y contiene las más altas aspiraciones de la gran mayoría del movimiento de personas en situación de discapacidad, en coherencia con el reconocimiento esencial de su "igual humanidad" (Urien, 2017). Finalmente, lo que consideramos muy importante es que esos documentos nos indican que hoy el derecho a la educación debe interpretarse, sin ambigüedades, como el derecho a la educación inclusiva.

Por lo que respecta al área de Educación del citado plan, se diseñó una estructura de evaluación mixta, con dos técnicas de recogida de información:

- Una consulta directa mediante cuestionarios a profesionales del ámbito educativo, así como a entidades vinculadas al tercer sector en la atención a personas con discapacidad.

- Una consulta a personas expertas en el ámbito de la educación inclusiva a través de la técnica Delphi.

Este texto se centra exclusivamente en los análisis y valoraciones generados como resultado de la técnica Delphi utilizada. Está prevista la difusión de los resultados derivados del cuestionario en otra publicación.

\subsection{Participantes}

La técnica Delphi utilizada en este trabajo se ha llevado a cabo con seis expertos y expertas $^{4}$ en educación. En un primer momento se invitó a participar a ocho personas, de las cuales dos de ellas, por diferentes motivos, no pudieron implicarse en el estudio. Las personas que han participado cuentan con una amplia y acreditada trayectoria profesional, de gestión e investigación en el ámbito de las personas en situación de discapacidad.

Se trata de cuatro mujeres y dos hombres con una media aproximada de veinticinco años de experiencia profesional que cubre ámbitos relacionados con el desarrollo y la educación de personas (niños y niñas, adolescentes, jóvenes y adultas) consideradas con discapacidades intelectuales o del desarrollo, con trastornos del espectro autista o con sordera o déficit auditivo, así como otros desarrollos atípicos. Entre ellos y ellas hay quien han trabajado en organizaciones del movimiento asociativo de las personas en situación de discapacidad, así como otras han ocupado puestos de alta dirección, gestión y supervisión de políticas educativas en materia de "educación especial” y "atención a la diversidad" en diferentes administraciones educativas de España. En este grupo también hay quienes trabajan habitualmente en tareas de asesoramiento y orientación psicopedagógica al profesorado y en la atención a familias. Por ello, las personas consultadas conocen bien el movimiento asociativo de las personas en situación de discapacidad en la CM y las normas internacionales, nacionales y locales que afectan a esta área.

4 Climent Giné, Manuel Ávila, Inés de Araoz, Marian Valmaseda, Juana María Hernández, María Luisa Galán. 
ANÁLISIS Y VALORACIÓN DEL ÁREA DE EDUCACIÓN DEL III PLAN DE ACCIÓN PARA PERSONAS CON DISCAPACIDAD EN LA COMUNIDAD DE MADRID (2012-2015)

G. ECHEITA, C. SIMÓN, C. MÁRQUEZ, M. ${ }^{a}$ L. DE LOS M. FERNÁNDEZ, E. PÉREZ Y A. MORENO

\subsection{Instrumento y procedimiento}

Como se ha mencionado anteriormente, para los fines del estudio se ha empleado la técnica Delphi, que es una herramienta utilizada especialmente en la evaluación de programas, en la toma de decisiones, así como en otras situaciones de análisis prospectivos o retrospectivos en los que, en la medida de lo posible, se pretende "poner de acuerdo" a personas expertas que participan y conocen bien un determinado ámbito o sector (León y Montero, 2015).

En esta técnica se aplica y analiza una secuencia de cuestiones a un grupo de participantes que se han seleccionado a partir de unos determinados criterios, relacionados con el objetivo y la temática del estudio que se lleva a cabo. El grupo coordinador proporciona una continua retroalimentación a las personas expertas y actúa como elemento dinamizador e integrador de los diferentes acuerdos (Varela-Ruiz, DíazBravo y García-Durán, 2012). Como señalan León y Montero (2015), se contemplan diferentes fases en su desarrollo, cuyo número puede variar en función, por ejemplo, del grado de acuerdo entre quienes han participado.

En todo caso, el procedimiento general es el siguiente: en una primera fase se formulan una serie de preguntas abiertas o en formato de cuestionario sobre el tema objeto de estudio. Una vez recibidas las respuestas a estas cuestiones se analizan por parte del equipo coordinador y, a partir de las mismas, se construye un segundo cuestionario o documento de síntesis que se remite a los participantes. Sea con un formato u otro, lo que se presenta es un conjunto de afirmaciones con la finalidad de que sean valoradas en cuanto a su grado de importancia y significación por el equipo de personas expertas. En todo caso, se pide que se argumenten por escrito las razones que han fundamentado las respuestas emitidas.

El análisis de estas respuestas permite la elaboración de un tercer cuestionario o documento de trabajo con las respuestas proporcionadas y con una ordenación o estructuración que les confiera significado y sentido y, en su caso, que permita establecer el grado de importancia relativa otorgada a todas o algunas de ellas. Una vez remitido de nuevo este tercer documento se les pide a los participantes que señalen el grado de acuerdo con los análisis y síntesis realizados. Si el consenso que se obtiene se considera adecuado, se da por concluido el proceso y se considera este último texto como informe final.

El grupo de participantes fue invitado formalmente a analizar el Área de Educación del PAPCDCM 12/15, remitiéndoles, junto con la carta de presentación e información, la siguiente documentación:

a) Documento oficial del Plan de Acción de las Personas con Discapacidad de la CM (2012-2015), con indicación de que se prestara especial atención al apartado específico relativo a "Educación", pero sin perder de vista que también había otros objetivos y actuaciones de carácter educativo en otros apartados del plan (programas horizontales), así como en el área de desarrollo especial: Trastornos del espectro autista y el área de Atención Temprana.

b) Borrador de la Observación o Comentario General sobre el Derecho a la Educación Inclusiva que el Comité de Derechos de las Personas con Discapacidad

(C) Ediciones Universidad de Salamanca / CC BY-NC-ND

Siglo Cero, vol. 48 (1), n. ${ }^{\circ}$ 261, 2017, enero-marzo, pp. 51-71 


\section{ANÁLISIS Y VALORACIÓN DEL ÁREA DE EDUCACIÓN DEL III PLAN DE ACCIÓN PARA PERSONAS CON DISCAPACIDAD EN LA COMUNIDAD DE MADRID (2012-2015) \\ G. ECHEITA, C. SIMÓN, C. MÁRQUEZ, M. ${ }^{a}$ L. DE LOS M. FERNÁNDEZ, E. PÉREZ Y A. MORENO}

había elaborado con anterioridad a la fecha de envío ${ }^{5}$ y que en esos momentos circulaba internacionalmente para su consulta, discusión y propuestas de mejora o de cambios.

Junto con otras explicaciones en dicha carta se explicitaban las preguntas directrices que debían guiar el análisis y valoración del plan en materia de Educación, formuladas de la siguiente forma:

1) Tomando en consideración la valoración que puedas realizar de las principales fortalezas y debilidades de este plan. ¿Consideras que el mismo ha podido tener, o no, un impacto positivo y significativo para el desarrollo del derecho a la educación inclusiva, en este caso, en la Comunidad de Madrid?

2) ¿Cuáles son, en tu opinión, los principales ámbitos de intervención y los objetivos generales que debería cubrir un plan para las Personas con Discapacidad en el Área de Educación que fuera coherente con la potenciación del derecho a la educación inclusiva tal y como hoy se plantea?

Préstese atención al hecho de que la segunda pregunta no tiene solo un carácter proyectivo -sobre "lo que debería ser"-, sino que también es una forma de reconocer implícitamente, en su caso, lo que no se ha planteado o realizado en este plan.

\subsection{Resultados}

Los resultados que se presentan a continuación a modo de informe de sintesis, elaborado por el equipo responsable de esta tarea en el proyecto MADINC, recogen la propuesta final de análisis y valoración consensuada y aceptada por los expertos y las expertas participantes correspondiente al Área de Educación'.

\subsubsection{Fortalezas del III Plan}

1. Se valora positivamente la existencia del PAPCDCM teniendo en cuenta que el mismo supone un reconocimiento del derecho que asiste a las personas en situación de discapacidad a que las administraciones pongan en marcha actuaciones dirigidas a reducir las situaciones de discriminación existentes y a promover medidas que avancen hacia la equiparación de oportunidades y la promoción de su calidad de vida. La existencia misma del plan es una oportunidad para evaluar sus resultados y

5 Junio de 2016. La citada Observación o Comentario General fue finalmente aprobada y difundida por el Comité de Derechos de las Personas con Discapacidad con fecha 1 de septiembre de 2016. Una traducción autorizada del mismo puede obtenerse en: http://sid.usal.es/idocs/F1/ACT56067/DerechoalaEducacionInclusivaArt24.pdf.

6 Ha de señalarse nuevamente que los análisis que se vierten en este Informe están referidos exclusivamente al Área de Educación y a las medidas educativas contempladas en algunos de los programas horizontales. No deben generalizarse, por lo tanto, a otras esferas o áreas del plan. 
ANÁLISIS Y VALORACIÓN DEL ÁREA DE EDUCACIÓN DEL III PLAN DE ACCIÓN PARA PERSONAS CON DISCAPACIDAD EN LA COMUNIDAD DE MADRID (2012-2015)

G. ECHEITA, C. SIMÓN, C. MÁRQUEZ, M. ${ }^{a}$ L. DE LOS M. FERNÁNDEZ, E. PÉREZ Y A. MORENO

rendir cuentas de los mismos, valorar las acciones emprendidas y realizar un ejercicio de prospectiva para el desarrollo de una educación más inclusiva.

2. Aunque no se ha entrado en el detalle del análisis del procedimiento mediante el cual se ha gestado, concretado y ejecutado el plan, también se valora positivamente el hecho de que el mismo haya sido una oportunidad de hacer efectivo el derecho de las personas en situación de discapacidad a participar activamente y ser escuchadas en los asuntos que les atañen.

3. Es bien sabido que el cumplimiento de los derechos de las personas en situación de discapacidad en cualquier de sus ámbitos es y será un proceso de larga duración (en cierta medida "interminable”), que demanda un esfuerzo sostenido y sostenible a largo plazo. En este sentido deben de ser bienvenidos los "pasos” que se den para seguir avanzando, si bien es cierto que estos pueden ser -como de hecho lo es "el paso" dado en este plan en materia de educación- muy insuficientes.

4. Ello no quita mérito ni valor al reconocimiento de bastantes acciones planteadas que han sido bienvenidas por sus destinatarios como aportes necesarios en la ingente tarea de consolidar un sistema educativo en la CM más inclusivo.

\subsubsection{Fundamentación}

5. Pero la mayor debilidad observada a este respecto no es que simplemente sea un plan insuficiente porque queden muchos objetivos y actuaciones que acometer, sino porque se cuestiona el planteamiento que fundamenta una buena parte de las actuaciones planteadas. Esto es, responden a una perspectiva o modelo que, a juicio de los expertos consultados, es propio de las prácticas de integración de primera generación y que, por ello, hace muy complicado el objetivo de promover y sostener, directa o indirectamente, el derecho a una educación inclusiva.

6. En este sentido existe la preocupación entre los y las expertas consultados de que lejos de facilitar el progreso hacia esa meta, lo planteado más bien lo inhiba u obstaculice, sin demérito, como se acaba de apuntar, de que algunas actuaciones específicas que contempla el plan puedan ser acertadas y positivas.

7. En el Área de Educación, se echa de menos una mayor fundamentación y coherencia, habida cuenta de la extensa base teórica, conceptual y de principios existentes en la esfera internacional, en materia de educación inclusiva proveniente de organismos internacionales solventes fuera de toda duda; partiendo de la ya lejana Declaración de Salamanca y su Marco de Acción sobre Necesidades Educativas Especiales (NEE) (UNESCO, 1994) ${ }^{7}$, hasta la más reciente Convención de la ONU sobre los Derechos de las Personas con Discapacidad (Naciones Unidas, 2006), pasando por las conclusiones y recomendaciones de la cuadragésima octava reunión del International Bureau of Education (IBE) y la UNESCO de 2008, desarrollada bajo el elocuente título de La educación inclusiva: el camino hacia el futuro ${ }^{8}$ o las

7 http://www.unesco.org/education/pdf/SALAMA_S.PDF.

8 http://www.ibe.unesco.org/fileadmin/user_upload/Policy_Dialogue/48th_ICE/CONFINTED_48-3_Spanish.pdf. 
que se encuentran en la Posición sobre los sistemas de educación inclusiva de la Agencia Europea para las Necesidades Especiales y la Educación Inclusiva9. Si a ello se añadiera el trabajo de la importante cantidad de personas expertas, así como del mundo académico y/o de la investigación de reconocido prestigio internacional y nacional, en ámbitos centrales para las finalidades y objetivos de plan (como los relacionados con el estudio de la Calidad de Vida, el Paradigma de Apoyos que se maneja en el ámbito de las personas con discapacidad intelectual o la iniciativa conocida como Respuesta a la Intervención-RTI en sus siglas en inglés-) ${ }^{10}$, se hace necesaria una revisión y justificación de la débil fundamentación conceptual del plan.

8. Ha de resaltarse el hecho de que en el propio plan -cuya articulación sobre el eje de los derechos es innegable, enmarcada en la referencia constante a la Convención de los Derechos de las Personas con Discapacidad (Naciones Unidas, 2006)solo aparezca una vez (p. 21) la referencia al art. 24 de la CDPCD que recoge el derecho a una educación inclusiva, y no de manera expresa sino de forma un tanto colateral respecto a su alcance más significativo ${ }^{11}$.

\subsubsection{Coherencia con la Convención sobre los Derechos de las personas con discapacidad}

9. Las personas consultadas han venido a resaltar la importancia y urgencia de un nuevo marco normativo que suponga un cambio real respecto a los enfoques y normas actuales (muchas de las todavía vigentes proceden de los años 90) ${ }^{12}$. Se trata de una tarea muy importante que debe acometerse desde el rigor y la coherencia con lo mucho que hoy se sabe al respecto y con la consideración como derecho de la educación inclusiva para el alumnado en situación de discapacidad.

10. En este sentido, un aspecto nuclear que no debe ser olvidado es que la CDPCD (reforzada con lo que se señala en el CG n. ${ }^{\circ} 4$, sobre el artículo 24 de la misma referido precisamente a la educación inclusiva) establece sin ambigüedades la inclusión educativa como la única modalidad educativa que debe seguirse en la escolarización del alumnado considerado en situación de discapacidad. En este sentido, mantener el planteamiento como el que recogía el Objetivo General del Programa 1 del PAPCDCM (Acceso al sistema educativo), que venía a establecer un ambiguo principio de inclusión "dentro de la modalidad más adecuada para cada alumno”, es incoherente con el principio general de inclusión (CG, punto

9 https://www.european-agency.org/.

10 https://www.understood.org/es-mx/school-learning/special-services/rti/understanding-response-to-intervention.

11 “... Se destaca la necesidad de trabajar para su educación en todos los niveles que permita desarrollar toda su potencialidad a las personas con discapacidad, algo que, por otra parte, redundará en el beneficio de toda la sociedad al incorporar los potenciales de estas personas a la comunidad (art. 24)”.

12 CM Orientación Educativa. NEE. Compilación de normativa vigente. http://www.madrid.org/cs/ Satellite?c=CM_InfPractica_FA\&cid=1142329685640\&idConsejeria=1109266187254\&idListConsj=1109 265444710\&idOrganismo=1142359974952\&language $=$ es\&pagename $=$ ComunidadMadrid $\% 2$ FEstructura \&pv $=1142329630823 \& \mathrm{sm}=1109266100977$. 
ANÁLISIS Y VALORACIÓN DEL ÁREA DE EDUCACIÓN DEL III PLAN DE ACCIÓN PARA PERSONAS CON DISCAPACIDAD EN LA COMUNIDAD DE MADRID (2012-2015)

G. ECHEITA, C. SIMÓN, C. MÁRQUEZ, M. ${ }^{a}$ L. DE LOS M. FERNÁNDEZ, E. PÉREZ Y A. MORENO

18) y considerado discriminatorio para los alumnos o alumnas que fueran escolarizados en tales centros (CG, 4 , punto 13 ).

11. Los informes, declaraciones y documentos internacionales en esta materia han puesto de manifiesto, reiteradamente, a la hora de planificar e implementar "planes de acción”, para los colectivos en riesgo de exclusión (como lo es el de las personas en situación de discapacidad), la necesidad imperiosa de articular tres planos interdependientes:

a. Un enfoque sistémico (referido al conjunto del sistema educativo, que conlleva hacer de la educación inclusiva un enfoque transversal a todos los elementos del mismo; ordenación, currículo, formación, financiación, evaluación, etc.);

b. Una perspectiva global (referida a la consideración de los centros escolares como unidad básica de actuación y que, por ello, debe incidir en sus culturas, sus políticas y en las prácticas que se llevan a cabo en las aulas), y

c. Una mirada integral respecto a las personas (que debe contemplar y respetar a los estudiantes en su singular diversidad de necesidades y estilos de aprendizaje y en sus múltiples inteligencias y competencias).

Tomado en consideración el marco anterior resulta difícilmente comprensible la ausencia del mismo en el PAPCDCM, enfoque que en el plan queda sustituido por un conjunto de actuaciones parciales, inconexas y de limitado alcance la mayoría de ellas.

12. Obviamente es comprensible que un plan trianual no pueda implementar todas las actuaciones necesarias en los múltiples planos que se acaban de apuntar. Pero es de esperar que las que acometa respondan a ese modelo complejo, sistémico e integral $\mathrm{y}$ a un proceso ordenado, secuenciado, coherente y sostenible a largo plazo.

\subsubsection{Estructura, objetivos y actuaciones}

13. Por otra parte, hay aspectos relativos a la estructura que sería conveniente considerar seriamente con vistas a futuros planes, pues la relación entre los objetivos generales (positivos en su conjunto, pero formulados de manera excesivamente amplia) y las actuaciones concretas previstas es muy mejorable (sin programas intermedios diferenciados), siendo estas últimas, o bien imprecisas en su significado y alcance (véase "garantizar la permanencia de los alumnos con discapacidad en el sistema educativo"), o bien, en otras muy insuficientes o reduccionistas, para considerar que de su sola aplicación se haya podido progresar significativamente hacia aquellos.

14. Se trata, por otra parte, de actuaciones que, en muchos casos (p. e., en lo referido a los compromisos con la dotación de equipos de FM para el alumnado con déficit auditivo o la firma de convenios para la presencia de intérpretes de Lengua de Signos Española en los centros escolares), ya existían y que no suponen ningún avance, sino, en el mejor de los casos, su continuidad.

15. Por otra parte, en la medida que las actuaciones más ambiciosas no tienen asociados indicadores precisos de consecución, se hace difícil una evaluación de impacto rigurosa de los mismos. 
ANÁLISIS Y VALORACIÓN DEL ÁREA DE EDUCACIÓN DEL III PLAN DE ACCIÓN PARA PERSONAS CON DISCAPACIDAD EN LA COMUNIDAD DE MADRID (2012-2015)

G. ECHEITA, C. SIMÓN, C. MÁRQUEZ, M. ${ }^{\text {a }}$ L. DE LOS M. FERNÁNDEZ, E. PÉREZ Y A. MORENO

16. Otro aspecto a considerar tiene que ver con el modelo de intervención que subyace y justifica algunas actuaciones. Las personas expertas consultadas parecen confluir en la valoración de que se trata de un conjunto de planteamientos y actuaciones que refuerzan una perspectiva individual, centrada en los servicios y asistencial (sobre todo, en las áreas de Atención Temprana y TEA).

17. A este modelo subyace fuertemente el criterio de que lo que se necesitan son "más recursos"; más o nuevo personal de apoyo para este alumnado (p. e., la figura del "integrador social" para los centros que escolarizan alumnado con TEA), nuevos servicios y centros (como el Centro Regional de Coordinación y Valoración Infantil, CRECOVI), así como más recursos tecnológicos para la habilitación y/o rehabilitación. Una forma de pensar que, a juicio de los expertos y expertas consultadas, está basada en la rehabilitación/corrección de problemas y que por ello tiende, a la larga, a reforzar el statu quo del sistema educativo en materia de educación inclusiva en lugar de promover su reforma y mejora constante.

18. Se trata de un enfoque que no está alineado con el modelo que subyace a los lineamientos que vienen señalando los organismos internacionales y las personas expertas en la materia y que, en esencia, apunta a la necesidad de implementar planes que confluyan en la necesidad de transformar el sistema educativo, mejorar los centros escolares (sus culturas y políticas de centro con una orientación inclusiva) e innovar respecto a las formas de enseñar y evaluar para que lo que finalmente se haga en la acción educativa que ocurre en las aulas (y otros espacios educativos) sea "asequible, accesible, aceptable y adaptable"13 a la diversidad de necesidades educativas del conjunto del alumnado.

\subsection{5. Ámbitos de actuación}

19. Desde este modelo "para la transformación y la innovación”, y sin pretender ser exhaustivos al respecto -toda vez que la literatura especializada disponible y los informes referidos ahondan sobradamente en estas cuestiones-, el grupo de expertos y expertas consultados han apuntado que los planes de esta naturaleza deberían incidir, entre otros muchos ámbitos, en los siguientes:

a. Establecimiento de una normativa clara y coherente con el significado y alcance que los organismos internacionales han dado a la "educación inclusiva".

b. Medidas de acceso a la educación (normativa de escolarización) y de accesibilidad (física, cognitiva, visual y auditiva) a los centros escolares (Escuelas de Educación Infantil y Primaria, CEIP, o Colegios e Institutos de Educación Secundaria Obligatoria) coherentes con el principio de inclusión.

c. Un currículo más reducido y compensado, de forma que permita trabajar equilibradamente todas las competencias imprescindibles, para incidir sobre todas las "inteligencias humanas", en particular las que tienen que ver con el equilibrio personal y las relaciones interpersonales (desarrollo emocional) y la convivencia.

13 Las “4 A del derecho a la educación”: https://revistas-colaboracion.juridicas.unam.mx/index.php/ rev-instituto-interamericano-dh/article/view/8220/7368. 
ANÁLISIS Y VALORACIÓN DEL ÁREA DE EDUCACIÓN DEL III PLAN DE ACCIÓN PARA PERSONAS CON DISCAPACIDAD EN LA COMUNIDAD DE MADRID (2012-2015)

G. ECHEITA, C. SIMÓN, C. MÁRQUEZ, M. ${ }^{\text {a }}$ L. DE LOS M. FERNÁNDEZ, E. PÉREZ Y A. MORENO

d. Formas de enseñar/aprender/evaluar diseñadas desde el principio para todos ("Diseño Universal de Aprendizaje") ${ }^{14}$ y con la capacidad de personalizar el aprendizaje de cada estudiante, con y sin discapacidad, como también recoge el CG n. ${ }^{\circ} 4$ del Comité de Derechos de las Personas con Discapacidad (p. 9).

e. Fortalecimiento de las "alianzas familia-escuela-profesionales", construyendo entre todos y en todos los implicados las capacidades clave para que ello sea posible: competencia profesional, comunicación, respeto, compromiso, equidad y confianza.

f. Formación inicial y permanente del profesorado ${ }^{15}$, para hacerle competente en tales formas de enseñar y para reforzar su alfabetización ética en relación con los valores y principios que sostienen el derecho a una educación inclusiva. Pero también es crítico y estratégico facilitar la formación y el desarrollo profesional desde una perspectiva inclusiva de otros agentes educativos concernidos en esta empresa: los equipos directivos como tales; los "especialistas" y el personal no docente y de servicios; los orientadores y orientadoras, y, de forma muy especial, los servicios de inspección.

g. Políticas sistemáticas y con carácter agregado de recopilación de evidencias que sean coherentes con la tarea de articular con equidad las oportunidades de acceder, participar y aprender de todos los alumnos y alumnas y, en este caso en particular, del alumnado considerado en situación de discapacidad. Recopilación de evidencias que debe realizarse tanto en los centros escolares como en los servicios de apoyo.

h. Liderazgo institucional entendido como con un liderazgo distribuido, gobernanza y autonomía educativa.

i. Políticas de desarrollo curricular (materiales, recursos, incentivos a la innovación, el intercambio de experiencias y la investigación educativa...) alineadas, por ejemplo, con principios básicos de la educación inclusiva; la accesibilidad y el diseño universal para el aprendizaje y la instrucción.

j. Financiación coberente con el objetivo de una educación inclusiva que haga posible el acceso, la permanencia y la promoción en el sistema educativo del alumnado en situación de discapacidad y, obviamente, la de cualquier otro estudiante en situación de vulnerabilidad.

\subsubsection{Algunas consideraciones sobre el Área de Atención Temprana}

20. Siendo el Área de Atención Temprana (AT) un ámbito estratégico para tratar de garantizar la igualdad de oportunidades de todos los niños y niñas, y muy en particular de quienes por razones diversas puedan verse insertos en contextos de

$14 \mathrm{http} / /$ www.educadua.es/doc/dua/dua_pautas_intro_cv.pdf.

15 No está de más recordar la baja incidencia en los Planes de Formación de la CM de las actuaciones formativas en materia de "Educación Especial, Compensatoria y Adultos", que en el curso 2014/15 se tradujeron en 55 cursos, con 459 matriculados y 416 certificados emitidos. En ese mismo período, el conjunto de actividades formativas fueron 2.019, con 35.072 matriculados y 30.408 certificados emitidos. Fuente: http://www.madrid.org/cs/. 
ANÁLISIS Y VALORACIÓN DEL ÁREA DE EDUCACIÓN DEL III PLAN DE ACCIÓN PARA PERSONAS CON DISCAPACIDAD EN LA COMUNIDAD DE MADRID (2012-2015)

G. ECHEITA, C. SIMÓN, C. MÁRQUEZ, M. ${ }^{a}$ L. DE LOS M. FERNÁNDEZ, E. PÉREZ Y A. MORENO

vulnerabilidad conviviendo con múltiples factores de riesgo a su alrededor, los expertos consultados también han hecho algunas consideraciones al respecto. Estas consideraciones giran, nuevamente, en relación con el enfoque o modelo que subyace a las intervenciones que se contemplan desde esta área.

21. A este respecto también han apreciado el refuerzo de un modelo médico/asistencial y de servicios de la Atención Temprana en el marco de una creciente privatización de dicha atención en la CM, que supone un grave perjuicio a las familias con menores recursos.

22. La creación del CRECOVI, un servicio centralizado para la coordinación y canalización de las demandas, no se considera una medida que vaya a mejorar realmente la AT. Hasta la fecha, por el contrario, han aumentado las listas de espera y el tiempo de demora para recibir dicha atención que, en algunas ocasiones, llega con un año de retraso respecto a la petición. En todo caso, una medida inicialmente positiva en la esfera de la coordinación interagencias (salud, educación, servicios sociales) parece que está teniendo resultados muy poco positivos al respecto, por lo que se antoja imprescindible con vistas al nuevo plan revisar a fondo su funcionamiento.

23. Se trata, por otra parte, de una actuación en dirección contraria a la que caminan otros países más avanzados en este dominio y que tiene que ver no tanto con la creación de nuevos servicios, como con potenciar el llamado enfoque o modelo centrado en la familia, con carácter universal, gratuito y de proximidad a la población, y cuya esencia es empoderar a las propias familias (mediante asesoramiento y formación en su propio contexto y alrededor de las rutinas infantiles). En definitiva, menos atención "asistencial” basada en el planteamiento de que las familias se desplacen a los centros de AT y más trabajo de capacitación de las familias para aprovechar las rutinas y relaciones cotidianas para reforzar el desarrollo infantil. Todo ello bajo criterios de equidad.

24. Sin duda, ello pasa por una reconsideración profunda del modelo de intervención, asesoramiento y apoyo de los diferentes centros y Equipos de Atención Temprana (EAT), algo que está ausente en este plan.

25. Por otra parte, este es un ámbito en el que las políticas dirigidas a la mejora real y efectiva de los planes de coordinación interagencias (salud, atención temprana, educación, bienestar social) es crítico para anticiparse y paliar los riesgos psicosociales asociados a la discapacidad en edades tempranas. Se trata de un hecho tantas veces reconocido como ignorado o débilmente planteado en la mayoría de los planes como el que nos ocupa; y este no es una excepción (salvo la iniciativa del CRECOVI ya comentada).

26. De hecho, en algunos aspectos no solo no se ha mejorado esta coordinación, sino que se ha debilitado. Tal es el caso de la ausencia de los orientadores/as en el Servicio de Atención a la Escolarización (SAE), que ahora ya no pueden aportar su conocimiento del sector en la tarea de alcanzar una escolarización equilibrada del alumnado más vulnerable en los distintos centros escolares, como venían haciendo hasta no hace mucho a través de las anteriores Comisiones de Escolarización de las distintas Áreas Territoriales. 
ANÁLISIS Y VALORACIÓN DEL ÁREA DE EDUCACIÓN DEL III PLAN DE ACCIÓN PARA PERSONAS CON DISCAPACIDAD EN LA COMUNIDAD DE MADRID (2012-2015)

G. ECHEITA, C. SIMÓN, C. MÁRQUEZ, M. ${ }^{a}$ L. DE LOS M. FERNÁNDEZ, E. PÉREZ Y A. MORENO

3.3.7. Algunas consideraciones en relación con el Área de desarrollo especial: Trastornos del Espectro Autista (TEA)

27. De momento tampoco están presentes (en forma de coordinación efectiva) los o las orientadoras del EOEP Específico de Alteraciones Graves del Desarrollo en la Unidad de Diagnóstico Complejo del Hospital Gregorio Marañón y centro Retiro. Siendo esta una iniciativa (AMI-TEA) que se valora como muy positiva en el ámbito de los TEA (por cuando redunda en la sensibilización del personal sanitario en lo referente a la atención a estas personas en el ámbito de la salud), es incomprensible que no estén presentes "profesionales educativos", pues la mejora de la calidad de vida de este alumnado no es solo cuestión de diagnóstico temprano, sino que también hay que valorar el funcionamiento global del niño y sus necesidades de apoyo en el ámbito educativo. Esta descoordinación va en detrimento de los alumnos con TEA y en aumento del trabajo y la tensión que supone a sus familias, en términos de nuevas derivaciones y demoras.

28. Esta coordinación interinstitucional es, sin duda, un asunto complejo, pero no menos que otros, y al mismo tiempo su mejora resulta crucial.

29. Hechas las consideraciones anteriores, que cabría adjetivar como de fondo, por cuanto se refieren a su fundamentación, a la calidad estructural del plan y al modelo de intervención para la mejora que, directa o indirectamente, desde el mismo se promueve, el grupo de expertos y expertas consultado también ha hecho apreciaciones más específicas que tienen que ver con elementos relevantes del sistema y con algunos de los grupos específicos de alumnos y alumnas considerados en situación de discapacidad.

30. Tal es el caso, en primer lugar, del alumnado considerado con Trastornos del Espectro Autista (TEA), cuya especial consideración en el plan es, cuando menos, llamativa puesto que para este grupo existe todo un Área de Desarrollo Especial en el mismo.

31. Sin duda, hay razones de peso que justifican este señalamiento particular y que enraízan, en primer lugar, con un pasado de mayor discriminación y desamparo que otros colectivos. Todo apunta, también, tanto a un importante incremento en la prevalencia de este trastorno del desarrollo (1 por cada 100 nacimientos), como a la mejora de las prácticas de detección y evaluación tempranas lo que, obviamente, ha servido para hacer visible la realidad y necesidades educativas de un alumnado que, en un pasado no muy lejano, tardaban en reconocerse y atenderse conforme a su derecho a una educación de calidad.

32. Por otra parte, nadie pone en duda que hablamos de un alumnado para quien el desafío frente a su derecho a una educación inclusiva resulta más difícil de cumplir que el de otros, habida cuenta no tanto de sus necesidades específicas de apoyo educativo (extensas y generalizadas en muchos casos), cuanto por las barreras a las que se enfrenta durante su escolarización en la mayoría de los centros y aulas ordinarias para alcanzar unos niveles justos de participación y aprendizaje.

33. Por estas y otras razones han sido y siguen siendo alumnos y alumnas frecuentemente escolarizados en CEE. 
ANÁLISIS Y VALORACIÓN DEL ÁREA DE EDUCACIÓN DEL III PLAN DE ACCIÓN PARA PERSONAS CON DISCAPACIDAD EN LA COMUNIDAD DE MADRID (2012-2015)

G. ECHEITA, C. SIMÓN, C. MÁRQUEZ, M. ${ }^{a}$ L. DE LOS M. FERNÁNDEZ, E. PÉREZ Y A. MORENO

34. En este contexto, el modelo de “Centros Preferentes” para la escolarización de este alumnado, que con frecuencia se reduce o malinterpreta como el modelo de "aulas preferentes" para este alumnado, ha sido fuertemente impulsado por la CM, pero sin duda alguna también por la petición expresa y reiterada de las familias implicadas y de muchos profesionales. Sea como fuere, ello ha sido un paso muy significativo en el proceso bacia su inclusión en los centros ordinarios y para poner en evidencia que también es posible su escolarización en este tipo de centros y, con ello, la de otros alumnos y alumnas con necesidades de apoyo igualmente extensas. Aunque no dudamos de la necesidad justificada de este paso, por sí solo es, a todas luces, insuficiente.

35. Sin duda, estar donde están los demás es el primer paso hacia el objetivo de cumplir el derecho a estar incluido. Pero este es mucho más que un asunto de localización. Conlleva el esfuerzo de transformar las culturas, las políticas y las prácticas escolares para articular con equidad dicha presencia, con las oportunidades de todo el alumnado de participar (ser valorado, reconocido, estimado) y aprender al máximo de sus potencialidades (que son muchas).

36. El problema no es "las aulas en sí", ni mucho menos, pues las mismas pueden configurarse como un verdadero recurso de apoyo a la escolarización inclusiva, y de hecho así ocurre en algunos casos muy ejemplarizantes que, por ejemplo, han implementado mejoras en las aulas, mejorado el tiempo y la convivencia en los patios o intensificada la colaboración con las familias, por señalar solo algunos aspectos. El problema está en la débil voluntad de muchos otros centros escolares a la hora de emprender el proceso de eliminar o minimizar las barreras existentes para la presencia, la participación y el aprendizaje de este alumnado en las aulas y espacios comunes.

37. A tenor de este criterio, los expertos consultados piden cautela con la política expansiva de estas "aulas preferentes" (que el plan contempla), toda vez que no se dispone de evidencias rigurosas y contrastadas sobre el impacto real de las mismas en términos de inclusión. $\mathrm{O}$, dicho de otra manera, la creación de 50 nuevas “aulas” no es una medida inclusiva en sí misma y, siendo una estrategia potencialmente valiosa, podrían estar generando actuaciones que simplemente mejoran la integración de este alumnado (su mera presencia en centros ordinarios), pero no significativamente su inclusión (presencia X participación X aprendizaje).

38. Se trataría, por otra parte, de aulas que tienen el grave riesgo de configurarse como "microcentros de educación especial", bastante aisladas, aunque sea en enclaves ordinarios, con un impacto limitado en la aspiración de que estos se transformen globalmente para dar cabida a experiencias y oportunidades más auténticas y más profundamente "inclusivas" no solo para el alumnado con TEA, sino para todos y todas.

39. Riesgo más que evidente cuando se observa, a juicio de los expertos y expertas consultadas, el pobre y limitado esquema seguido para mejorar la formación del profesorado de tales “centros preferentes” y la gran dosis de improvisación en el proceso de implementación de este programa.

40. Se observa con preocupación el desigual trato y las desiguales oportunidades que tiene el alumnado con o sin TEA, pero con otras necesidades de apoyo educativo 
ANÁLISIS Y VALORACIÓN DEL ÁREA DE EDUCACIÓN DEL III PLAN DE ACCIÓN PARA PERSONAS CON DISCAPACIDAD EN LA COMUNIDAD DE MADRID (2012-2015)

G. ECHEITA, C. SIMÓN, C. MÁRQUEZ, M. ${ }^{a}$ L. DE LOS M. FERNÁNDEZ, E. PÉREZ Y A. MORENO

específico. El derecho de estos alumnos a una educación inclusiva tiene que hacerse extensivo a otros alumnos que comparten necesidades y no crear agravios comparativos.

\subsubsection{Sobre los Centros de Educación Especial}

41. Con toda la inversión realizada en este terreno de los "Centros/aulas preferentes para la escolarización del alumnado con TEA”, es importante denunciar, como han hecho algunos de los expertos consultados, que en la CM siguen creciendo las plazas en Centros de Educación Especial (CEE) para este y otro alumnado con necesidades de apoyo extensas y generalizadas. En consecuencia, no se está garantizando el derecho de acceso y permanencia en el sistema educativo de las personas con TEA, dentro de un marco inclusivo, como se planteaba en el plan. $\mathrm{Ni}$ de estas ni de otras con similares necesidades de apoyo. En el curso 2013/14 el 22,5\% del alumnado considerado con necesidades educativas especiales estaba escolarizado en $\mathrm{CEE}^{16}$.

42. Al hilo de este dato, que no puede quedar como un asunto anecdótico o secundario, se señala como urgente, por parte de los expertos, que la CM aborde la cuestión de los CEE en el marco del derecho a una educación inclusiva. Ya en 1994, la UNESCO, con motivo de la Declaración de Salamanca y su Marco de Acción, instaba a los gobiernos y administraciones educativas a detener el crecimiento de este tipo de centros y a iniciar, cabría decir, sin prisas, pero sin calma, su proceso de transformación al servicio de una escolarización inclusiva para todo el alumnado, "incluidos aquellos con discapacidades más graves”, como se decía en dicho Informe (UNESCO, 1994, punto 10 del Marco de Acción).

43. Pero han pasado más de 22 años y, como se acaba de señalar, lejos de cumplir con ese compromiso firme y solemnemente asumido por el Gobierno español (y la $\mathrm{CM}$ en la parte alícuota que le corresponde), se avanza en la dirección contraria. Si observamos la evolución de la escolarización del alumnado considerado con necesidades educativas especiales en CEE, hay que denunciar que se ha producido un incremento en los últimos diez años (hasta el curso 2013/14) del 24,1\% ${ }^{17}$. El plan analizado no contemplaba en absoluto esta situación y ningún plan futuro será creíble y digno de consideración si no acomete esta empresa con seriedad y rigor.

\subsubsection{Cuestiones relacionadas con la evaluación psicopedagógica y los apoyos}

44. El caso del alumnado con TEA da pie para poner en evidencia dos importantes aspectos que le afectan negativamente, tanto a este, en particular, como al conjunto del alumnado considerado con $N E E$ en general. Nos referimos a los

16 http://www.madrid.org/bvirtual/BVCM016247.pdf.

17 P. 252 del informe: http://www.madrid.org/cs/Satellite?c=CM_Actuaciones_FA\&cid=11425555 49082\&idConsejeria $=1109266187254 \& i d L i s t C o n s j=1109265444710 \& i d O r g a n i s m o=1109266227481 \& p a g$ ename $=$ ComunidadMadrid $\% 2 F E$ structura . 
ANÁLISIS Y VALORACIÓN DEL ÁREA DE EDUCACIÓN DEL III PLAN DE ACCIÓN PARA PERSONAS CON DISCAPACIDAD EN LA COMUNIDAD DE MADRID (2012-2015)

G. ECHEITA, C. SIMÓN, C. MÁRQUEZ, M. ${ }^{a}$ L. DE LOS M. FERNÁNDEZ, E. PÉREZ Y A. MORENO

procesos de evaluación psicopedagógica y a los relativos a la organización de los apoyos escolares.

45. El modelo y las prácticas de evaluación psicopedagógica existente y los procedimientos administrativos asociados a los procesos de escolarización del alumnado considerado con NEE (dictamen del EOEP, comisión de escolarización, Resolución de la Administración), están anclados y son devotos de un planteamiento anterior a la Convención de los Derechos de las Personas con Discapacidad. Los mismos parten del convencimiento de que "puede haber alumnos o alumnas no integrables en centros ordinarios” y, en consecuencia, ordenan sus actuaciones a diferenciar unos y otros (quiénes pueden integrarse y quiénes tienen que estar en otra modalidad educativa), a sustentar técnicamente la decisión (dictamen del EOEP) y a concretarla en una resolución administrativa que cierra el proceso: centro ordinario, aula de educación especial, centro de educación especial.

46. Los expertos y expertas consultados coinciden plenamente en que este enfoque es contrario al art. 24 de la CDPCD y, por lo tanto, urgen a que se transforme en consonancia con lo allí dispuesto. En todo caso, el PAPCDCM nada dice ni propone al respecto, configurando con ello una debilidad muy relevante toda vez que el mismo cubre un período (2012/15) en el que hacía cuatro años que el Gobierno de España había ratificado la Convención (2008).

47. A este respecto hay que hacer especial mención al trabajo de los Equipos de Orientación Educativa y Psicopedagógica (de Atención Temprana, Generales y Específicos) que, por sus funciones y tareas habituales, tienen un papel relevante en este proceso de escolarización, así como en el seguimiento educativo de este alumnado. Un papel que, en todo caso, tiene que cambiar y reformularse conforme a las implicaciones del desarrollo del derecho a una educación inclusiva. En este nuevo marco ya no tiene sentido que buena parte de su tiempo, esfuerzo y dedicación esté concentrado en la tarea de evaluación y "diagnóstico” de las necesidades educativas especiales (que categoriza al alumnado por sus carencias y dificultades y olvida sus fortalezas) y en toda la parafernalia administrativa que lo acompaña (dictámenes y resoluciones de escolarización). En un escenario educativo que hace de la inclusión su marco de referencia, su trabajo está llamado a desarrollarse, fundamentalmente, alrededor de las funciones de asesoramiento y apoyo a la mejora e innovación educativa para que todo el alumnado (sin restricciones), pueda participar y aprender. Un cambio que no llegará si no va acompañado, entre otras consideraciones, de una nueva estructura y modelo organizativo, de planes de formación adecuados y de una nueva normativa específica que sustituya la vigente, que se remonta a mediados de los años noventa.

48. No deja de ser sorprendente que los EOEPS específicos sobre discapacidad (sobre alumnado sordo/discapacidad auditiva, con ceguera/discapacidad visual, con TEA o con discapacidades motoras) no hayan sido singularmente consultados en la elaboración de un plan de esta naturaleza, al menos, en el Área de Educación.

49. Las políticas de integración escolar han estado acompañadas de un "falso amigo”, relativo a la dotación extraordinaria de recursos personales de apoyo (sobre todo profesorado con las especialidades de Pedagogía Terapéutica y Audición y

(C) Ediciones Universidad de Salamanca / CC BY-NC-ND

Siglo Cero, vol. 48 (1), n. ${ }^{\circ}$ 261, 2017, enero-marzo, pp. 51-71

$$
-67-
$$


ANÁLISIS Y VALORACIÓN DEL ÁREA DE EDUCACIÓN DEL III PLAN DE ACCIÓN PARA PERSONAS CON DISCAPACIDAD EN LA COMUNIDAD DE MADRID (2012-2015)

G. ECHEITA, C. SIMÓN, C. MÁRQUEZ, M. ${ }^{a}$ L. DE LOS M. FERNÁNDEZ, E. PÉREZ Y A. MORENO

Lenguaje). Los expertos lo han llamado "falso amigo" porque parece natural y lógico que el desafío de la escolarización de alumnos con especiales necesidades de apoyo educativo vaya acompañado de dicha dotación extra. Pero el tiempo pasado y las investigaciones disponibles al respecto han puesto de manifiesto que este esquema ha resultado ser, en efecto, un "falso amigo", pues con su presencia e incorporación en los centros, lejos de ayudar y contribuir a la transformación, enriquecimiento e innovación de las prácticas educativas comunes, su trabajo se ha traducido, en muchísimas ocasiones, en "hacerse cargo y responsabilizarse, en buena medida, de ese alumnado”. En definitiva, dar respuesta justa a las necesidades educativas del alumnado más vulnerable, parece ser, en muchos casos, tarea "de otros", pero no el desafío de un proyecto educativo colegiado.

50. No es de extrañar que, en este contexto y con esta mentalidad, la demanda más fuertemente reiterada cuando se consulta al profesorado y a las familias sobre las necesidades para mejorar los procesos de inclusión haya sido clara y rotunda: más apoyos, más especialistas, más orientadores, mejores ratios alumno/especialistas, etc. Por otra parte, como se ha apuntado, la propia CM a través de la normativa establecida sobre "apoyos” ha venido a reforzar este enfoque de los apoyos centrados en la "atención al alumnado".

51. Ninguno de los expertos consultados niega la necesidad de mejorar la dotación de los centros escolares en términos de profesorado y “apoyos”, mucho más tras años de recortes brutales en cuanto a su número, justificados por la "crisis económica”. Se necesitan más recursos personales (y materiales) pero, una vez más, claramente orientados y dirigidos a la transformación y mejora de la capacidad de los centros en su conjunto y del profesorado en sus aulas, para articular con equidad las oportunidades de estar, participar y aprender de todo el alumnado, sin exclusiones. El plan se ha quedado muy lejos de este planteamiento y, como se viene comentando, más bien ha apoyado con algunas de sus actuaciones la situación que se debe cambiar.

\subsubsection{Apuntes sobre algunas actuaciones necesarias}

52. Al señalar los aspectos mejorables o con mayor debilidad del Plan 12/15 los expertos han coincidido en reconocer que su reverso configuraría un conjunto de líneas de intervención, programas y actuaciones que necesariamente han de contemplarse por la CM, antes o después, si realmente se quiere avanzar hacia un sistema educativo madrileño más inclusivo. Con ello daban por contestado lo fundamental de la segunda pregunta planteada en esta consulta.

53. No obstante, también han señalado otros aspectos o actuaciones que son igualmente necesarios, pero sin la intención por su parte de ser exhaustivos ni en su enumeración ni en su explicación, habida cuenta de que el encargo recibido no lo permitía. A continuación, se indican algunos de ellos sin que su orden signifique prelación o importancia:

a. La preocupación por el llamativo aumento de las situaciones de maltrato entre iguales por abuso de poder en los centros escolares (particularmente en la etapa 
de la educación secundaria) tiene que tener una respuesta educativa integral y preventiva de mayor calado y consistencia de la que hasta la fecha parece estar ofreciéndose para el conjunto del alumnado. Pero a este respecto no hay que olvidar que la situación de discapacidad es un factor de riesgo en estos procesos y que, por lo tanto, por razones de justicia, es urgente anticiparse a los acontecimientos reforzando en los planes y programas generales de prevención y actuación, medidas y acciones específicas para el alumnado en situación de discapacidad. Las expertas y expertos consultados se han mostrado muy preocupados por este asunto y aquellos que conocen más de cerca la realidad de los centros madrileños han echado en falta en las iniciativas puestas en marcha (no tanto en lo previsto en el plan) esta sensibilidad hacia este grupo de alumnos y alumnas en particular.

b. El asunto de los planes y programas para la transición a la vida adulta (TVA) de los estudiantes en situación de discapacidad es una tarea que ha de acometerse muy seriamente en el marco de los Planes de Orientación Educativa, Académica y Profesional en particular de los centros que imparten educación secundaria (obligatoria y bachillerato) y/o formación profesional. Este es un asunto que habitualmente solo se entiende y se trata en el marco de las actuaciones de los CEE, pero es hora de tomar conciencia de que es una pieza clave en el proceso de una educación más inclusiva hacia una vida adulta igualmente inclusiva. A este respecto se ha apuntado que el programa "ACOMPAÑADOS” al que hace referencia el plan tiene poco de inclusivo nuevamente habida cuenta de un enfoque "experto" donde son otros los que analizan y toman las decisiones sobre "lo que conviene" a los jóvenes considerados con discapacidad. Un planteamiento que se encuentra alejado de los trabajos de Planificación Centrada en la Persona (PCP) que se vienen impulsando desde el movimiento asociativo de las personas en situación de discapacidad (particularmente en relación con las discapacidades intelectuales y del desarrollo ${ }^{18}$.

c. En relación con el ámbito educativo, pero en la esfera de los servicios sociales y deportivos comunitarios, hay que implementar planes y políticas específicas para mejorar el disfrute del tiempo libre y el acceso al ocio, el deporte y la cultura de los jóvenes en situación de discapacidad. Hay que estar muy vigilantes ante las situaciones de exclusión de las actividades complementarias en los centros escolares de estos estudiantes.

d. Hoy más que nunca y mañana todavía más, se hará evidente que la formación no empieza y termina en los contextos escolares, sino que se prolonga a lo largo de la vida adulta, en consonancia con el enfoque del LLL (LifeLong Learning). En este sentido las actuaciones educativas para las personas adultas en situación de discapacidad tienen que ser igualmente objeto de una consideración mucho más profunda en los futuros planes de lo que ha sido en este.

e. La situación de discapacidad es para muchos alumnos y alumnas un factor de riesgo en cuanto al desarrollo de su salud mental. Se han echado en falta iniciativas que aborden esta cuestión en el marco de las acciones de coordinación

18 http://www.plenainclusionmadrid.org/grupo/uds-de-planificacion-centrada-en-la-persona-pcp/. 
interinstitucional e interagencias anteriormente comentadas. A este respecto no hay que perder de vista que la salud mental y determinados "problemas de conducta” son más una cuestión educativa que psiquiátrica.

f. Con relación al alumnado sordo o con discapacidad auditiva, también se han apuntado algunas cuestiones importantes, que tienen mucho que ver con una significativa falta de recursos: por ejemplo, solo hay un Centro de Atención Temprana especializado en las necesidades de este alumnado; las dotaciones de nuevos equipos de FM están llegando a tardar más de un año; se constata una progresiva pérdida de identidad de algunos centros preferentes al no contar con profesionales formados en la atención educativa a este alumnado; además, la falta de transporte escolar en algunas localidades afecta directamente a la posibilidad de recibir la respuesta educativa que este alumnado requiere.

g. Se ha señalado con gran preocupación la pobre y limitada oferta formativa para el alumnado en situación de discapacidad en relación con los estudios de formación profesional (básica, media o superior).

h. Por lo que respecta a la promoción de la educación inclusiva en la etapa de los estudios universitarios, se ha reiterado que el modelo y los planes de actuación deben seguir el mismo enfoque y principios establecidos para la educación obligatoria. Sin duda hay algunos aspectos singulares dentro de la misma que deberían ser objeto de atención, como la accesibilidad en todas sus dimensiones (p. e., física, cognitiva, visual, auditiva); las dificultades que encuentra este alumnado a la hora de ajustar su vocación o intereses a las posibilidades de “inclusión” que ofrecen algunos campus; el esfuerzo importante que algunos deben hacer en el ámbito de las relaciones sociales, o todo lo relativo a la mejora de su empleabilidad (p. e., en relación con la adquisición y manejo de un segundo idioma) y de su transición al empleo.

i. En todo caso, hoy se sabe que el factor clave para el acceso a la Universidad de los estudiantes considerados con discapacidad está antes de llegar a la prueba de acceso a la misma o a los propios campus universitarios: está en la etapa de Bachillerato o Formación Profesional. Algunos analistas lo vienen a considerar como una auténtica "travesía del desierto" pues, durante estas etapas, estos estudiantes parecen "perder todos sus derechos” y buena parte de los apoyos disponibles en la educación obligatoria. Sin una intervención profunda en la mejora de la inclusión educativa también en esas etapas, seguiremos observando y manteniendo el actual porcentaje desproporcionadamente bajo de estudiantes en situación de discapacidad que acceden a la Universidad.

j. Finalmente se ha señalado que el proceso hacia una educación más inclusiva requiere del apoyo y la comprensión constante de la sociedad. Hay que vencer muchos prejuicios y estereotipos todavía fuertemente establecidos en la población adulta sobre las supuestas implicaciones negativas de la inclusión del alumnado con discapacidad para el desarrollo educativo de sus compañeros y compañeras sin discapacidad. Por ese motivo, es importante, en expresión de una de las expertas consultadas, "poner de moda la inclusión” con amplias, 
inteligentes y bien diseñadas campañas de divulgación, promoción y reconocimiento de los valores y principios que sostienen esta meta, así como de los efectos positivos para todos que se pueden conseguir con su implementación.

54. A tenor de sus análisis y evaluaciones finalmente se les pedía a los expertos y expertas consultadas una valoración cualitativa global sobre el previsible grado de impacto que este plan haya podido tener en términos de mejora de la educación de las personas con discapacidad de la CM. Su valoración ha sido unánime: en el mejor de los casos, muy reducido/limitado.

\section{Referencias bibliográficas}

León, O. y Montero, I. (2015). Métodos de investigación en Psicología y Educación (4. ${ }^{\text {ed.). }}$. Madrid: McGraw-Hill.

Naciones Unidas (2006). Convención de los derechos de laspersonas con discapacidad. Consultado en: http://www.un.org/spanish/disabilities/default.asp?id=497. Recuperado el 15/ 02/2016.

Naciones Unidas. CRPD (2016). General comment N. ${ }^{\circ}$ (2016). Article 24: Right to inclusive education. Convention on the Rights of Persons with Disabilities. Recuperado de http:// www.ohchr.org/EN/HRBodies/CRPD/Pages/CRPDIndex.aspx. Traducción de M. Á. Verdugo y J. M. Amor en: http://sid.usal.es/idocs/F1/ACT56067/DerechoalaEducacionInclusivaArt24.pdf.

Schalock, R. y Verdugo, M. Á. (2007). El concepto de calidad de vida en los servicios y apoyos para personas con discapacidad intelectual. Siglo Cero, 38 (4), 21-36.

Urien, T. (2017). Del reconocimiento legal al reconocimiento efectivo. De la igual dignidad como un derecho de la persona con discapacidad intelectual o del desarrollo. Un proceso que nos interpela. Siglo Cero, 47 (2), 43-62.

Varela-Ruiz, M., Díaz-Bravo, L. y García-Durán, R. (2012). Descripción y usos del método Delphi en investigaciones del área de la salud. Investigación en Educación Médica, 1 (2), 90-95. 